\title{
1-辛基-3-甲基咪唑离子液体作为可循环溶剂萃取/去除水中二价录研究
}

\author{
王晓伟 $b, c \quad$ 陈莎*,,$b$ \\ ( ${ }^{a}$ 北京工业大学 区域大气复合污染防治北京市重点实验室 北京 100124) \\ $\left(^{b}\right.$ 北京工业大学 环境与能源工程学院 北京 100124) \\ ( ${ }^{c}$ 北京市疾病预防控制中心与北京市预防医学研究中心 食物中毒诊断溯源技术北京市重点实验室 北京 100013)
}

\begin{abstract}
摘要 本研究采用 1-辛基-3-甲基咪唑离子液体 $\left(\left[\mathrm{C}_{8} \mathrm{MIM}\right] \mathrm{PF}_{6}\right.$ )建立了水中 $\mathrm{Hg}^{2+}$ 的循环去除方法. 首先使用 $\left[\mathrm{C}_{8} \mathrm{MIM}\right] \mathrm{PF}_{6}$ 萃取水中 $\mathrm{Hg}^{2+}$, 随后通过甲酸的还原反应, 去除萃取到 $\left[\mathrm{C}_{8} \mathrm{MIM}\right] \mathrm{PF}_{6}$ 中的 $\mathrm{Hg}^{2+}$, 进而实现 $\left[\mathrm{C}_{8} \mathrm{MIM}\right] \mathrm{PF}_{6}$ 的回收与循环使 用. 本研究优化了萃取与还原去除条件, 考察了最佳条件下 $\left[\mathrm{C}_{8} \mathrm{MIM}\right] \mathrm{PF}_{6}$ 的循环使用能力. 结果表明, $50 \mathrm{~mL}$ 水中加入 1 $\mathrm{mL}\left[\mathrm{C}_{8} \mathrm{MIM}\right] \mathrm{PF}_{6}$ 同时加入 $0.2 \mathrm{~mL}$ 1-甲基咪唑, $50{ }^{\circ} \mathrm{C} 、 220 \mathrm{r} / \mathrm{min}$ 震荡 $2 \mathrm{~h}$, 对 $\mathrm{Hg}^{2+}$ 的萃取效率接近 $100 \%$. 随后在离子液 体中加入 $4 \mathrm{~mL}, 40 \%$ 甲酸溶液, $50{ }^{\circ} \mathrm{C}$ 下 $220 \mathrm{r} / \mathrm{min}$ 震荡 $30 \mathrm{~min}$, 可以将 $\left[\mathrm{C}_{8} \mathrm{MIM}\right] \mathrm{PF}_{6}$ 中 $60 \% \sim 70 \%$ 的 $\mathrm{Hg}^{2+}$ 还原去除. 采用 这一方式对水中 $\mathrm{Hg}^{2+}$ 进行循环萃取, 在 9 次萃取中, $\left[\mathrm{C}_{8} \mathrm{MIM}^{\mathrm{M}} \mathrm{PF}_{6}\right.$ 对 $\mathrm{Hg}^{2+}$ 的去除效率保持在 $83 \% \sim 98 \%$. 因此, 本方法 不仅实现 $\left[\mathrm{C}_{8} \mathrm{MIM}\right] \mathrm{PF}_{6}$ 对水中 $\mathrm{Hg}^{2+}$ 的去除，同时实现了 $\left[\mathrm{C}_{8} \mathrm{MIM}\right] \mathrm{PF}_{6}$ 的回收与循环使用，避免了 $\left[\mathrm{C}_{8} \mathrm{MIM}\right] \mathrm{PF}_{6}$ 过度使用所 带来的环境问题.
\end{abstract}

关键词＼cjkstart离子液体; 录离子; 液-液萃取; 还原; 甲酸

\section{Use of Ionic Liquid 1-Octyl-3-methylimidazolium Hexafluorophosphate as a Recyclable Solvent for Extraction/Removal of $\mathrm{Hg}$ (II) from Water

\author{
Wang, Xiaowei ${ }^{b, c} \quad$ Chen, Sha*,a,b
} \\ ( ${ }^{a}$ Key Laboratory of Beijing on Regional Air Pollution Control, Beijing University of Technology, Beijing 100124, China) \\ ( ${ }^{b}$ College of Environmental and Energy Engineering, Beijing University of Technology, Beijing 100124, China) \\ ( ${ }^{c}$ Beijing Key Laboratory of Diagnostic and Traceability Technologies for Food Poisoning, Beijing Centers for Diseases Control and Prevention \& Centers for Preventive Medical Research, Beijing 100013, China)}

\begin{abstract}
Mercury, a highly persistent and toxic substance, seriously threatens the human health and the environment. As an environmental friend solvent, ionic liquids (ILs) have been used as replacements for conventional organic solvents in various separation processes. But there are still some technical aspects that need to be addressed before ILs can be practically applied to remove $\mathrm{Hg}$ (II) from water. The first and most important issue is the recovery and recycling of ILs without significant loss to make the process economically viable and reduce their potential environmental burden. In this study, a novel approach for removal of $\mathrm{Hg}(\mathrm{II})$ from water was developed via 1-octyl-3-methylimidazolium hexafluorophosphate $\left(\left[\mathrm{C}_{8} \mathrm{MIM}_{\mathrm{I}} \mathrm{PF}_{6}\right) . \mathrm{Hg}(\mathrm{II})\right.$ was extracted into $\left[\mathrm{C}_{8} \mathrm{MIM}\right] \mathrm{PF}_{6}$ and then reduced into $\mathrm{Hg}(0)$ with formic acid. Extraction and reduction conditions were optimized. Because the extraction efficiency of $\mathrm{Hg}(\mathrm{II})$ into the pristine $\left[\mathrm{C}_{8} \mathrm{MIM}\right] \mathrm{PF}_{6}$ was limited, $0.4 \%(V / V)$ 1-methylimidazole was added as complex agent of $\mathrm{Hg}(\mathrm{II})$ into $\left[\mathrm{C}_{8} \mathrm{MIM}\right] \mathrm{PF}_{6}$ to enhance the extraction efficiency. Under a phase ratio of $\left[\mathrm{C}_{8} \mathrm{MIM}\right] \mathrm{PF}_{6}$ to water of $1: 50$, almost $100 \%$ of the $\mathrm{Hg}(\mathrm{II})$ in water phase $(1 \mathrm{mg} / \mathrm{L})$ was extracted into the $\left[\mathrm{C}_{8} \mathrm{MIM} \mathrm{PF}_{6}\right.$ phase after shaking for $2 \mathrm{~h}$ at $220 \mathrm{r} / \mathrm{min}$ and $50{ }^{\circ} \mathrm{C}$. Afterwards, $\mathrm{Hg}(\mathrm{II})$ concentrated in the IL was reduced to $\mathrm{Hg}(0)$ by formic acid under the heating condition, and then the $\left[\mathrm{C}_{8} \mathrm{MIM}\right] \mathrm{PF}_{6}$ was isolated for the purpose of recycling the IL. The $\mathrm{Hg}(\mathrm{II})$ in [ $\left.\mathrm{C}_{8} \mathrm{MIM}\right] \mathrm{PF}_{6}$ was reduced to $\mathrm{Hg}(0)$ using $40 \%(\mathrm{~V} / \mathrm{V})$ formic acid at $50{ }^{\circ} \mathrm{C}$. Under a phase ratio of $40 \%(\mathrm{~V} / \mathrm{V})$ formic acid to $\left[\mathrm{C}_{8} \mathrm{MIM}\right] \mathrm{PF}_{6}$ of $4: 1$, about $60 \% \sim 70 \%$ of $\mathrm{Hg}(\mathrm{II})$ was reduced to $\mathrm{Hg}(0)$ in 20 min. The formed $\mathrm{Hg}(0)$ in the mixture was removed after centrifugation, and the $\left[\mathrm{C}_{8} \mathrm{MIM}\right] \mathrm{PF}_{6}$ was collected and recycled. The extraction efficiency for $\mathrm{Hg}(\mathrm{II})$ in water was $c a .83 \%$ in the initial two runs, and then increased to $91 \% \sim 98 \%$ in the following 7 runs by the attribution of the formic acid which is residues in the IL phase and enhances extraction efficiency.

Keywords ionic liquid; mercury; liquid-liquid extraction; reduction; formic acid
\end{abstract}

* E-mail: chensha@bjut.edu.cn; Tel.: 0086-010-67391659; Fax: 0086-010-67391983

Received August 15, 2014; published September 30, 2014.

Project supported by the National Natural Science Foundation of China (No. 31301474) and the Public Service Applications of Beijing Centers for Preventive Medical Research (No. 2014-BJYJ-12).

项目受国家自然科学基金(No. 31301474)和北京市预防医学研究中心公益应用课题(No. 2014-BJYJ-12)资助. 


\section{1 引言}

录(Mercury, $\mathrm{Hg}$ ) 是一种具有高度持久性与生物蓄 积性的有害物质, 严重威胁着环境与人类健康 ${ }^{[1,2]} \cdot \mathrm{Hg}^{0}$ 极易挥发, 可以进入大气环流实现长距离传输与分布 ${ }^{[3]}$. $\mathrm{Hg}^{2+}$ 具有较高的细胞毒性, 同时 $\mathrm{Hg}^{2+}$ 与多种无机离子、 有机化合物, 尤其是与含有硫的化合物具有较强的亲和 作用 ${ }^{[4]}$. 目前, Hg 不仅是美国 EPA 优先控制污染物名单 中 13 种金属元素之一, 同时也被列入欧盟水框架指令 中地表水优先控制污染物名单. 因此, 地表水中录污染 的防控与去除具有重要意义.

目前，基于离子交换、表面吸附或离子的络合作用， 多孔二氧化硅 ${ }^{[5]}$ 、炭质吸附剂 ${ }^{[6,7]}$ 以及磁性纳米材料 ${ }^{[8]}$ 等 众多材料已经被用来去除水中的 $\mathrm{Hg}^{2+}$. 离子液体(Ionic Liquids, ILs)作为一种完全由离子组成的盐, 常温下呈 液态. 由于其具有众多优异的化学性能, 目前已被尝试 用来取代传统的液-液萃取过程中的有机溶剂. 虽然有 研究报道在不添加络合剂的条件下, $\mathrm{Hg}^{2+}$ 可以被烷基咪 唑类 ILs 萃取 ${ }^{[9,10]}$, 但是亦有研究认为这种直接萃取方 式对 $\mathrm{Hg}^{2+}$ 的萃取能力较差 ${ }^{[11]}$, 在萃取过程中需要加入 杯芳烃类化合物 ${ }^{[12]}$ 或双硫腙 ${ }^{[13]}$ 等络合配体以提高萃取 效率. 为了简化萃取过程, 更多研究开始关注将特定基 团修饰到离子液体上，使离子液体同时具有溶剂与络合 剂的功能 ${ }^{[9,11,14,15]}$.

虽然众多研究认为, ILs 将在污水处理方面发挥重 要作用, 但是目前仍有众多技术问题需要考察与解决. 其中最为重要的问题就是萃取完成后, ILs 中 $\mathrm{Hg}^{2+}$ 的去 除与 ILs 的回收与循环使用, 解决这一问题可以显著降 低污水处理过程的成本, 同时降低 ILs 过度排入环境所 带来的潜在环境问题. 目前已报道多种有效的还原 $\mathrm{Hg}^{2+}$ 的方法, 包括 $\mathrm{TiO}_{2}$ 光催化法 ${ }^{[16]}$ 、超声还原法 ${ }^{[17]}$ 、甲酸还 原法 ${ }^{[18]}$ 、加热还原法 ${ }^{[19]}$ 等. 本研究认为可以在萃取完成 后, 通过将 ILs 中的 $\mathrm{Hg}^{2+}$ 还原为单质录, 实现 ILs 与录 的分离与循环使用.

因此, 本研究首先考察了 1-辛基-3-甲基咪唑离子 液体 $\left(\left[\mathrm{C}_{8} \mathrm{MIM}\right] \mathrm{PF}_{6}\right)$ 萃取去除水中 $\mathrm{Hg}^{2+}$ 的能力, 优化萃取 条件以获得稳定、高效的萃取效率. 在此基础上, 进一 步考察萃取后 ILs 中 $\mathrm{Hg}^{2+}$ 的还原去除方式, 实现 ILs 的 回收与重复使用.

\section{2 结果与讨论}

\section{1 水中 $\mathrm{Hg}^{2+}$ 萃取条件优化}

本研究选择使用 $\left[\mathrm{C}_{8} \mathrm{MIM}\right] \mathrm{PF}_{6}$ 离子液体萃取水中 $\mathrm{Hg}^{2+}$, 分别考察了萃取温度、萃取时间、离子液体及络 合试剂的用量对萃取效率的影响.

图 1(a)表明, [ $\left.\mathrm{C}_{8} \mathrm{MIM}\right] \mathrm{PF}_{6}$ 对 $\mathrm{Hg}^{2+}$ 的萃取效率随萃取 温度的升高而显著提高, 萃取温度 $90{ }^{\circ} \mathrm{C}$ 时, 萃取效率 比 $20{ }^{\circ} \mathrm{C}$ 时提高 $20 \%$. 这主要是由于当温度提高时,

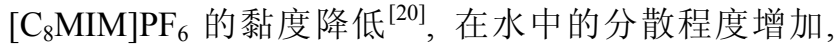
有利于 $\mathrm{Hg}^{2+}$ 萃取效率提高.

同时，整个萃取过程可以在短时间内达到平衡，由 图 1(b) 可知, 经过 $10 \mathrm{~min}$ 萃取后达到最高值 70\%. 但是, 当不添加络合剂使用 $\left[\mathrm{C}_{8} \mathrm{MIM}\right] \mathrm{PF}_{6}$ 直接萃取时, $\left[\mathrm{C}_{8} \mathrm{MIM}\right] \mathrm{PF}_{6}$ 的用量对萃取效率与萃取速度的影响明显 [图 1(c)], 当使用 $10 \%(V / V)\left[\mathrm{C}_{8} \mathrm{MIM} \mathrm{PF}_{6}\right.$ 萃取时, 可在 0.5 $\mathrm{h}$ 内萃取 $75 \%$ 的 $\mathrm{Hg}^{2+}$, 在 $2 \mathrm{~h}$ 内萃取 $80 \%$ 的 $\mathrm{Hg}^{2+}$, 当 $\left[\mathrm{C}_{8} \mathrm{MIM}\right] \mathrm{PF}_{6}$ 用量降低到 $4 \%$ 时, 达到平衡时只能萃取 $40 \%$ 的 $\mathrm{Hg}^{2+}$. 如果继续降低 $\left[\mathrm{C}_{8} \mathrm{MIM}\right] \mathrm{PF}_{6}$ 用量到 $2 \%$ 时, 经过 $3 \mathrm{~h}$ 萃取, 萃取效率缓慢提高到 $20 \%$.

由于金属离子与咪唑类 ILs 亲水性的差异, 咪唑类 ILs 本身对金属离子的萃取能力较差, 需要加入特定络 合剂与金属离子形成络合物以改变金属离子的亲水性, 提高萃取效率 ${ }^{[21]}$. 考虑到 1-甲基咪唑既可以与 $\mathrm{Hg}^{2+}$ 形 成络合物 ${ }^{[22]}$, 又是合成 $\left[\mathrm{C}_{8} \mathrm{MIM}\right] \mathrm{PF}_{6}$ 的原料之一, 结合 相似相溶原理, 我们推测在萃取过程中加入一定量的 1甲基咪唑可以有效提高 $\left[\mathrm{C}_{8} \mathrm{MIM}\right] \mathrm{PF}_{6}$ 对 $\mathrm{Hg}^{2+}$ 的萃取效率. 由图 1(d) 可知 $\left[\mathrm{C}_{8} \mathrm{MIM}\right] \mathrm{PF}_{6}$ 对 $\mathrm{Hg}^{2+}$ 的萃取效率随着 1-甲 基咪唑的加入显著提高, 尤其是在 $\left[\mathrm{C}_{8} \mathrm{MIM}\right] \mathrm{PF}_{6}$ 用量较 低的条件下. 当萃取体系中 $\left[\mathrm{C}_{8} \mathrm{MIM}\right] \mathrm{PF}_{6}$ 浓度为 $2 \%$ 时, 保持 1-甲基咪唑的浓度为 $0.4 \%(V / V)$, 即可获得与使用 $10 \%\left[\mathrm{C}_{8} \mathrm{MIM}\right] \mathrm{PF}_{6}$ 萃取时相当的萃取效率. 同时, 这一 结果也可以用来解释已有研究中对于烷基咪唑类 ILs 对 $\mathrm{Hg}^{2+}$ 萃取能力的差异. 这一差异可能主要由不同批次与 纯度的 ILs 中残留的 1-甲基咪唑的浓度差异所导致 ${ }^{[0 \sim 13]}$.

\section{$2.2\left[\mathrm{C}_{8} \mathrm{MIM}_{\mathrm{P}} \mathrm{PF}_{6}\right.$ 中 $\mathrm{Hg}^{2+}$ 还原去除条件优化}

随后, 本研究采用甲酸还原法对 $\left[\mathrm{C}_{8} \mathrm{MIM}\right] \mathrm{PF}_{6}$ 中 $\mathrm{Hg}^{2+}$ 进行还原去除, 分别对还原过程中使用的甲酸溶液浓 度、反应温度、反应时间以及甲酸溶液体积进行了优化.

试验结果表明, $\left[\mathrm{C}_{8} \mathrm{MIM}\right] \mathrm{PF}_{6}$ 中 $\mathrm{Hg}^{2+}$ 的还原效率随 着甲酸浓度的提高而线性增加[图 2(a)]. 但是, 高浓度 的甲酸溶液会导致 $\left[\mathrm{C}_{8} \mathrm{MIM}\right] \mathrm{PF}_{6}$ 的溶解，使 $\left[\mathrm{C}_{8} \mathrm{MIM}\right] \mathrm{PF}_{6}$ 无法循环使用. 尤其是当甲酸浓度超过 $70 \%(V / V)$ 时, $\left[\mathrm{C}_{8} \mathrm{MIM}\right] \mathrm{PF}_{6}$ 完全溶解, 无法进行分相. 因此, 本研究选 取 $20 \%, 40 \%, 60 \%$ 三个甲酸浓度对其还原条件进行考 察.

随着反应温度的逐渐提高, 不同浓度的甲酸溶液对 $\left[\mathrm{C}_{8} \mathrm{MIM}\right] \mathrm{PF}_{6}$ 中 $\mathrm{Hg}^{2+}$ 的还原效率出现明显差异 [图 2(b)]. 使用 $20 \%$ 的甲酸溶液, 还原效率随着温度的提高线性增 加; 使用 $40 \%$ 的甲酸溶液, 还原效率在反应温度 $50 \sim 70$ ${ }^{\circ} \mathrm{C}$ 时达到最大值; 而使用 $60 \%$ 的甲酸溶液，还原效率在 $50{ }^{\circ} \mathrm{C}$ 时达到最大值, 继续提高温度还原效率降低, 同 时出现离子液体溶解现象.

此外, $\left[\mathrm{C}_{8} \mathrm{MIM}\right] \mathrm{PF}_{6}$ 中 $\mathrm{Hg}^{2+}$ 的还原过程可以在短时 间内达到平衡[图 2(c)]. 同时随着所使用甲酸溶液体积 的增加, $\mathrm{Hg}^{2+}$ 的还原效率逐渐增加 [图 2(d)]. 当甲酸溶液 与 $\left[\mathrm{C}_{8} \mathrm{MIM}\right] \mathrm{PF}_{6}$ 的体积比为 $4: 1$ 时, 约 $60 \% \sim 70 \%$ 的 


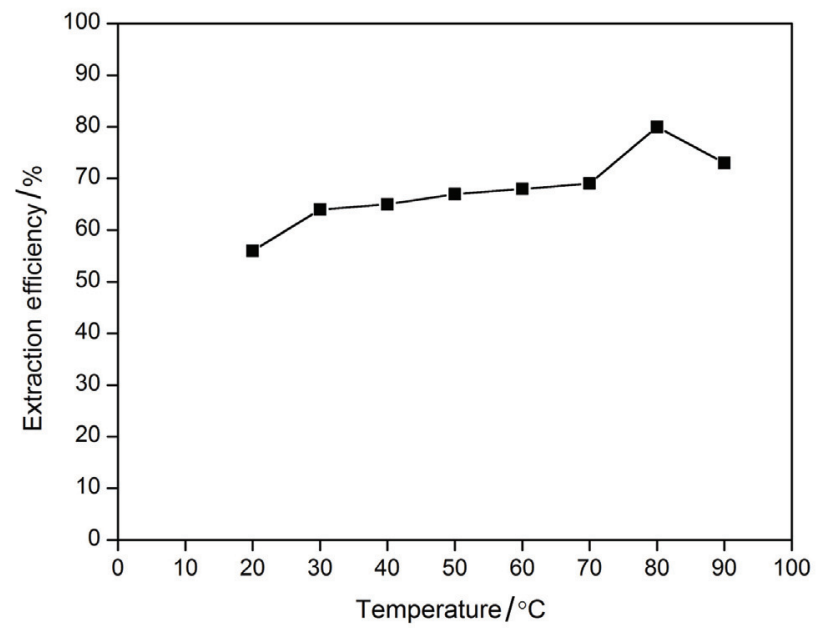

(a)

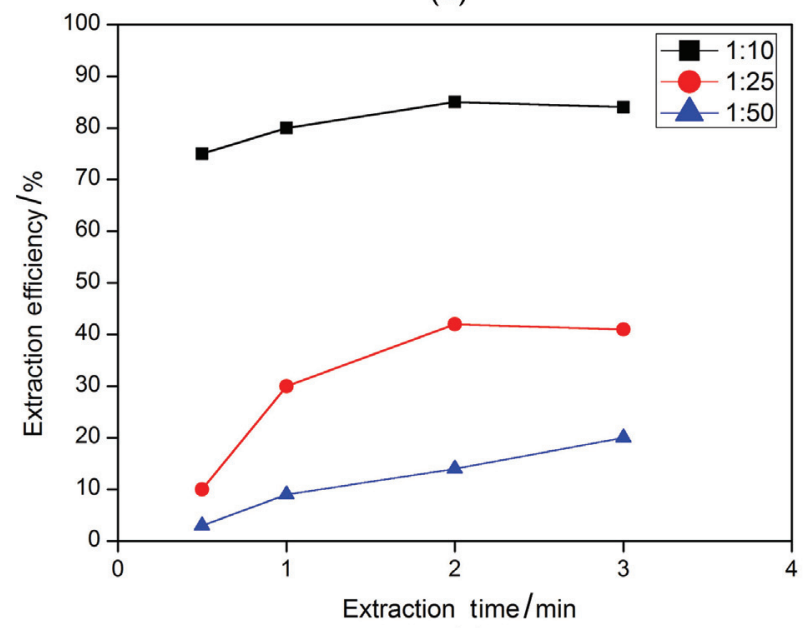

(c)

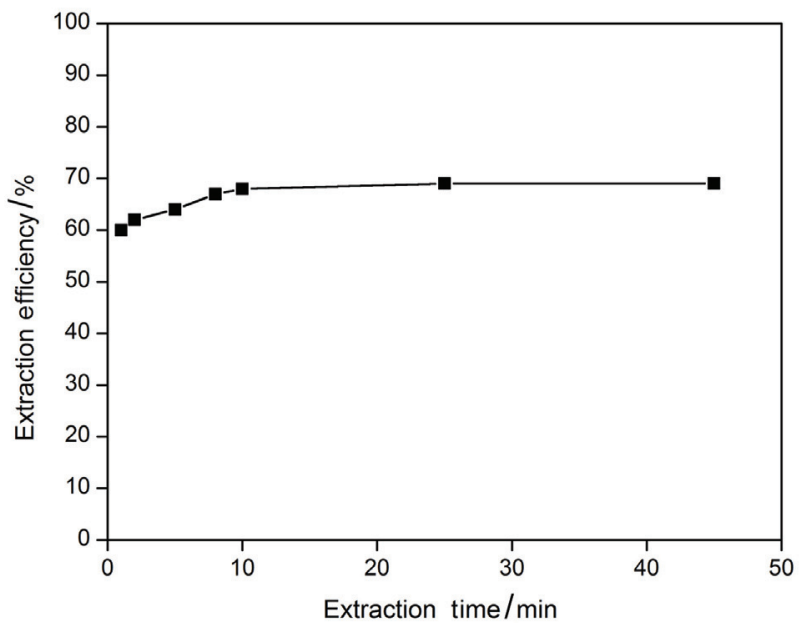

(b)

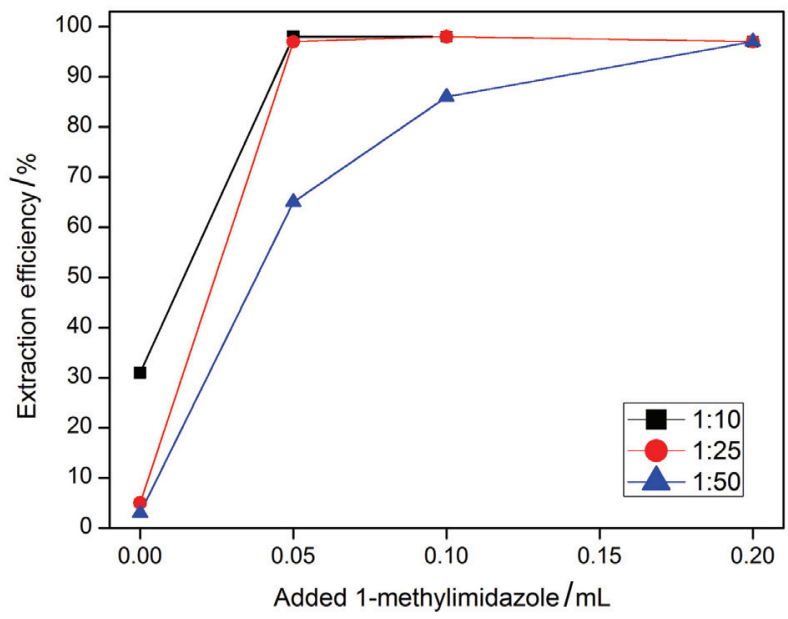

(d)

图 1 萃取条件优化: (a)萃取温度: $1 \mathrm{~mL} \mathrm{Hg}^{2+}$ 水溶液 $\left(1000 \mathrm{mg} / \mathrm{L}\right.$ )中加入 $1 \mathrm{~mL}\left[\mathrm{C}_{8} \mathrm{MIM}\right] \mathrm{PF}_{6}, 220 \mathrm{r} / \mathrm{min}$ 震荡 $30 \mathrm{~min}$; (b)萃取时间: $1 \mathrm{~mL} \mathrm{Hg}^{2+}$ 水溶液 $(1000 \mathrm{mg} / \mathrm{L})$ 中加入 $1 \mathrm{~mL}\left[\mathrm{C}_{8} \mathrm{MIM}\right] \mathrm{PF}_{6}, 50{ }^{\circ} \mathrm{C}, 220 \mathrm{r} / \mathrm{min}$ 震荡; (c) $\left[\mathrm{C}_{8} \mathrm{MIM}\right] \mathrm{PF}_{6}$ 用量: 分别在 $10,25,50 \mathrm{~mL} \mathrm{Hg}^{2+}$ 水溶液 $(1 \mathrm{mg} / \mathrm{L})$ 中加入 $1 \mathrm{~mL}$ $\left[\mathrm{C}_{8} \mathrm{MIM}\right] \mathrm{PF}_{6}, 50{ }^{\circ} \mathrm{C}, 220 \mathrm{r} / \mathrm{min}$ 震荡; (d)络合剂(1-甲基咪唑)的影响: 分别在 $10,25,50 \mathrm{~mL} \mathrm{Hg}{ }^{2+}$ 水溶液 $\left(1 \mathrm{mg} / \mathrm{L}\right.$ )中加入 $1 \mathrm{~mL}\left[\mathrm{C}_{8} \mathrm{MIM} \mathrm{PF}_{6}\right.$, 同时加入 不同体积 1-甲基咪唑, $50{ }^{\circ} \mathrm{C}, 220 \mathrm{r} / \mathrm{min}$ 震荡 $2 \mathrm{~h}$

Figure 1 Optimization of extraction condition: (a) Extraction temperature: Into $1 \mathrm{~mL}, 1000 \mathrm{mg} / \mathrm{L} \mathrm{Hg}$ (II) solution was added $1 \mathrm{~mL}\left[\mathrm{C}_{8} \mathrm{MIM} \mathrm{PF}_{6}\right.$, and the

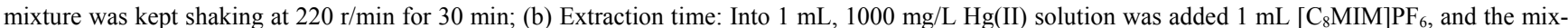
ture was kept at $50{ }^{\circ} \mathrm{C}$ and shaken at $220 \mathrm{r} / \mathrm{min}$; (c) Phase ratio: Into 10,25 and $50 \mathrm{~mL} 1 \mathrm{mg} / \mathrm{L} \mathrm{Hg}$ (II) solutions, respectively, was added $1 \mathrm{~mL}$ of $\left[\mathrm{C}_{8} \mathrm{MIM} \mathrm{PF}_{6}\right.$, and the mixtures were kept at $50{ }^{\circ} \mathrm{C}$ and shaken at $220 \mathrm{r} / \mathrm{min}$; (d) Effect of complexant (1-methylimidazole): Into $10,25 \mathrm{and} 50 \mathrm{~mL} 1 \mathrm{mg} / \mathrm{L}$ $\mathrm{Hg}$ (II) solutions, respectively, was added $1 \mathrm{~mL}\left[\mathrm{C}_{8} \mathrm{MIM}\right] \mathrm{PF}_{6}$ and different volumes 1-methylimidazole, and the mixture were kept at $50{ }^{\circ} \mathrm{C}$ and shaken at $220 \mathrm{r} / \mathrm{min}$ for $2 \mathrm{~h}$

$\mathrm{Hg}^{2+}$ 被还原去除. 由于 $40 \%$ 与 $60 \%$ 的甲酸溶液对 $\mathrm{Hg}^{2+}$ 的还原效率差异较小，同时考虑到甲酸溶液过多导致 $\left[\mathrm{C}_{8} \mathrm{MIM}\right] \mathrm{PF}_{6}$ 消耗增加, 因此, 最终选择 $40 \%$ 的甲酸溶 液, 甲酸溶液与 $\left[\mathrm{C}_{8} \mathrm{MIM}\right] \mathrm{PF}_{6}$ 的体积比为 $2: 1$.

\section{$2.3\left[\mathrm{C}_{8} \mathrm{MIM}_{\mathrm{P}} \mathrm{PF}_{6}\right.$ 离子液体的循环使用}

最后, 本研究考察了在最佳萃取与还原条件下, 使 用 $\left[\mathrm{C}_{8} \mathrm{MIM}\right] \mathrm{PF}_{6}$ 对水中 $\mathrm{Hg}^{2+}$ 进行循环去除的能力. 使用 $\left[\mathrm{C}_{8} \mathrm{MIM}\right] \mathrm{PF}_{6}$ 对 $\mathrm{Hg}^{2+}$ 浓度为 $1 \mathrm{mg} / \mathrm{L}$ 的水溶液进行萃取, 随后使用甲酸溶液对 $\left[\mathrm{C}_{8} \mathrm{MIM}\right] \mathrm{PF}_{6}$ 中的 $\mathrm{Hg}^{2+}$ 进行还原去 除, 最后将 $\left[\mathrm{C}_{8} \mathrm{MIM}\right] \mathrm{PF}_{6}$ 与甲酸溶液离心分离, 继续进行 下一循环萃取. 结果如图 3 所示, 在循环萃取开始时, 萃取效率约为 $85 \%$, 随着循环的进行, 萃取效率小幅度
提高且保持 $95 \%$ 的萃取率.

\section{3 结论}

本研究基于 $\left[\mathrm{C}_{8} \mathrm{MIM}\right] \mathrm{PF}_{6}$, 建立了水中 $\mathrm{Hg}^{2+}$ 的萃取 与还原去除方法. 使用 1-甲基咪唑作为络合剂, 可以显 著提高 $\left[\mathrm{C}_{8} \mathrm{MIM}\right] \mathrm{PF}_{6}$ 对 $\mathrm{Hg}^{2+}$ 的萃取能力; 同时通过甲酸 对 $\mathrm{Hg}^{2+}$ 的还原反应, 可以快速、便捷的将萃取到 $\left[\mathrm{C}_{8} \mathrm{MIM}\right] \mathrm{PF}_{6}$ 中的 $\mathrm{Hg}^{2+}$ 还原去除. 使用该方法可以实现 $\left[\mathrm{C}_{8} \mathrm{MIM}\right] \mathrm{PF}_{6}$ 对水中 $\mathrm{Hg}^{2+}$ 的循环去除, 减少了萃取过程 中 $\left[\mathrm{C}_{8} \mathrm{MIM}\right] \mathrm{PF}_{6}$ 的消耗，降低了 $\left[\mathrm{C}_{8} \mathrm{MIM}\right] \mathrm{PF}_{6}$ 排放产生的 二次污染问题，是一种经济有效、环境友好的 $\mathrm{Hg}^{2+}$ 去除 方法. 


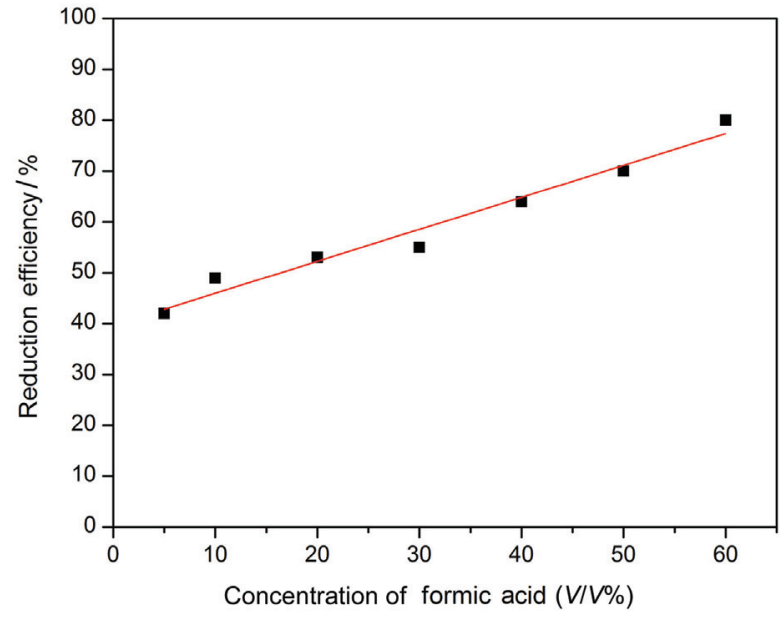

(a)

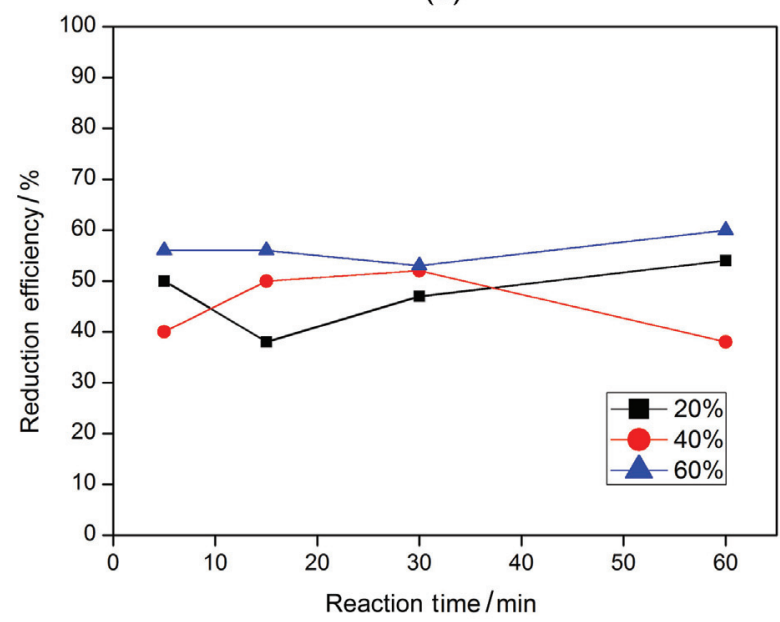

(c)

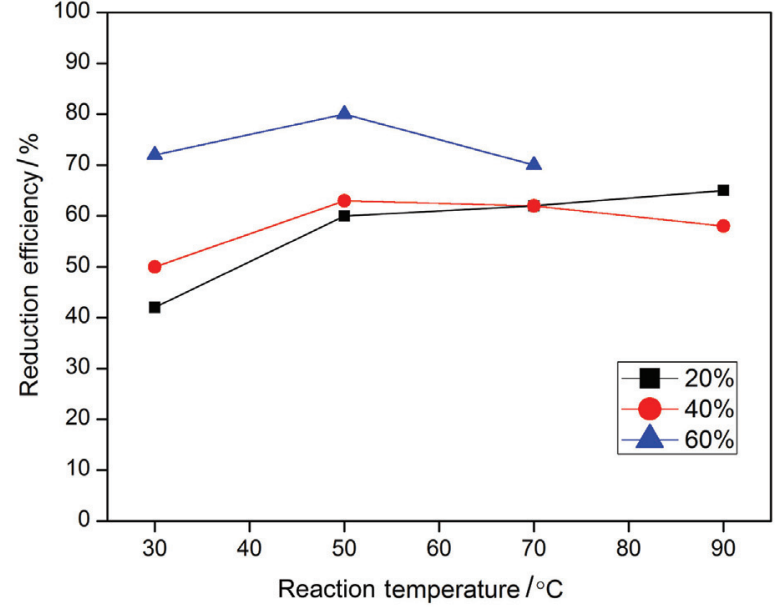

(b)

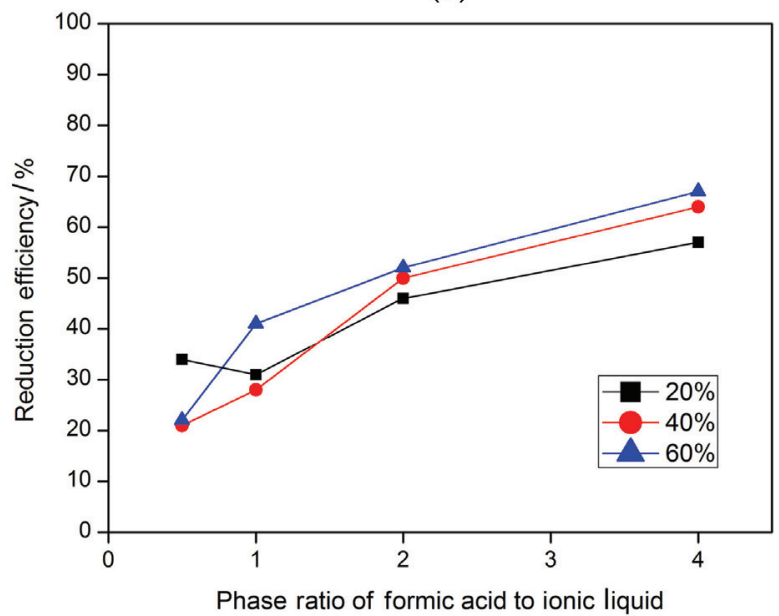

(d)

图 $2\left[\mathrm{C}_{8} \mathrm{MIM}^{-} \mathrm{PF}_{6}\right.$ 中 $\mathrm{Hg}^{2+}$ 还原条件优化: (a) 甲酸溶液浓度: $1 \mathrm{~mL}$ 含有 $1 \mathrm{mg} / \mathrm{L} \mathrm{Hg}^{2+}$ 的 $\left[\mathrm{C}_{8} \mathrm{MIM}\right] \mathrm{PF}_{6}$ 中加入 $2 \mathrm{~mL}$ 不同浓度甲酸溶液, $50{ }^{\circ} \mathrm{C}, 220 \mathrm{r} / \mathrm{min}^{\circ}$ 震荡 $30 \mathrm{~min}$; (b)还原温度: $1 \mathrm{~mL}$ 含有 $1 \mathrm{mg} / \mathrm{L} \mathrm{Hg}^{2+}$ 的 $\left[\mathrm{C}_{8} \mathrm{MIM}\right] \mathrm{PF}_{6}$ 中加入 $2 \mathrm{~mL}$ 浓度为 $20 \%, 40 \%, 60 \%$ 甲酸溶液, $220 \mathrm{r} / \mathrm{min}$ 震荡 $30 \mathrm{~min}$; (c)还原时间: $1 \mathrm{~mL}$ 含有 $1 \mathrm{mg} / \mathrm{L} \mathrm{Hg}^{2+}$ 的 $\left[\mathrm{C}_{8} \mathrm{MIM}\right] \mathrm{PF}_{6}$ 中加入 $2 \mathrm{~mL}$ 浓度为 $20 \%, 40 \%, 60 \%$ 甲酸溶液, $50{ }^{\circ} \mathrm{C}, 220 \mathrm{r} / \mathrm{min}$ 震荡; (d) 甲酸溶液用量: $1 \mathrm{~mL}$ 含有 $1 \mathrm{mg} / \mathrm{L}$ $\mathrm{Hg}^{2+}$ 的 $\left[\mathrm{C}_{8} \mathrm{MIM}\right] \mathrm{PF}_{6}$ 中加入不同体积浓度为 $20 \%, 40 \%, 60 \%$ 甲酸溶液, $50{ }^{\circ} \mathrm{C}, 220 \mathrm{r} / \mathrm{min}$ 震荡 $30 \mathrm{~min}$

Figure 2 Optimization of reduction condition: (a) Concentration of formic acid solution: Into $1 \mathrm{~mL}\left[\mathrm{C}_{8} \mathrm{MIM}\right] \mathrm{PF}_{6}$ containing $1 \mathrm{mg} / \mathrm{L} \mathrm{Hg}(\mathrm{II}) \mathrm{mas}$ added 2 $\mathrm{mL}$ formic acid solution which has various concentration, and the mixture was shaken at $50{ }^{\circ} \mathrm{C}$ for $30 \mathrm{~min}$; (b) Reaction temperature: Into $1 \mathrm{~mL}$ [ $\mathrm{C}_{8} \mathrm{MIM}_{\mathrm{PF}}$ containing $1 \mathrm{mg} / \mathrm{L} \mathrm{Hg}(\mathrm{II})$ were added $2 \mathrm{~mL}$ of $20 \%, 40 \%$ and $60 \%$ formic acid solution, respectively, and the mixture shaken for $30 \mathrm{~min}$; (c) Reaction time: Into $1 \mathrm{~mL}\left[\mathrm{C}_{8} \mathrm{MIM}\right] \mathrm{PF}_{6}$ containing $1 \mathrm{mg} / \mathrm{L} \mathrm{Hg}(\mathrm{II})$ were added $2 \mathrm{~mL}$ of $20 \%, 40 \%$ and $60 \%$ formic acid solution, respectively, the mixture shaken at $50{ }^{\circ} \mathrm{C}$; (d) Phase ratio: Into $1 \mathrm{~mL}\left[\mathrm{C}_{8} \mathrm{MIM}_{\mathrm{PF}}\right.$ containing $1 \mathrm{mg} / \mathrm{L} \mathrm{Hg}(\mathrm{II})$ were added $20 \%, 40 \%$ and $60 \%$ formic acid solution at varied phase ratio, respectively, and the mixture was shaken at $50{ }^{\circ} \mathrm{C}$ for $30 \mathrm{~min}$

\section{4 实验部分}

\section{1 主要试剂与仪器}

原子荧光分光光度计购自北京瑞利分析仪器公司. 梅特勒 AL204 分析天平, IKA MS3 数字混匀器.

试验所用 $\left[\mathrm{C}_{8} \mathrm{MIM}\right] \mathrm{PF}_{6}$ 离子液体购自上海易利生化 试剂有限公司. 称取适量氯化永(购自默克)溶于 $5 \%$ $(V / V) \mathrm{HNO}_{3}$ 中, 配置 $1000 \mathrm{mg} / \mathrm{mL} \mathrm{Hg}^{2+}$ 储备液. 每次使 用前, 使用 $5 \%(V / V) \mathrm{HNO}_{3}$ 稀释, 配置工作溶液. $\mathrm{Hg}^{2+}$ 与 甲酸溶液于每次实验前配置. 甲酸、1-甲基咪唑及其它 试剂均购自西格玛.

\section{2 试验方法}

萃取过程在 $15 \mathrm{~mL}$ 玻璃离心管中进行, [ $\mathrm{C}_{8} \mathrm{MIM} \mathrm{PF}_{6}$ 与 $\mathrm{Hg}^{2+}$ 溶液经过加热震荡后, 冷却至室温后, 2000 $\mathrm{r} / \mathrm{min}$ 离心 $10 \mathrm{~min}$, 吸出上层水溶液, 使用冷蒸气发生原子荧光光谱法测定其中 $\mathrm{Hg}^{2+}$ 浓度，计算萃取效率. 随 后在 $15 \mathrm{~mL}$ 玻璃离心管中加入甲酸溶液, 加热震荡, 冷 却至室温后, $2000 \mathrm{r} / \mathrm{min}$ 离心 $10 \mathrm{~min}$, 吸出上层甲酸溶 液.

需要注意, 在还原过程中, 需要等待离心管冷却后 再进行离心分离, 使 $\mathrm{Hg}^{0}$ 沉淀到离心管底部, 避免其中 $\mathrm{Hg}^{0}$ 蒸汽挥发对人身体健康产生危害. 
$\left[\mathrm{C}_{8} \mathrm{MIM}\right] \mathrm{PF}_{6}$ 中 $\mathrm{Hg}^{2+}$ 浓度测定: 通过反萃取法测定 下层 $\left[\mathrm{C}_{8} \mathrm{MIM}\right] \mathrm{PF}_{6}$ 剩余 $\mathrm{Hg}^{2+}$. 向 $15 \mathrm{~mL}$ 玻璃离心管中加 入 $5 \mathrm{~mL}$ 半胱氨酸溶液 $(1 \%, m / V), 3 \mathrm{~g} \mathrm{NaCl}, 60{ }^{\circ} \mathrm{C}$ 震荡 2 $\mathrm{h}$, 随后冷却至室温离心分离, 测定上层溶液中 $\mathrm{Hg}^{2+}$ 浓 度.

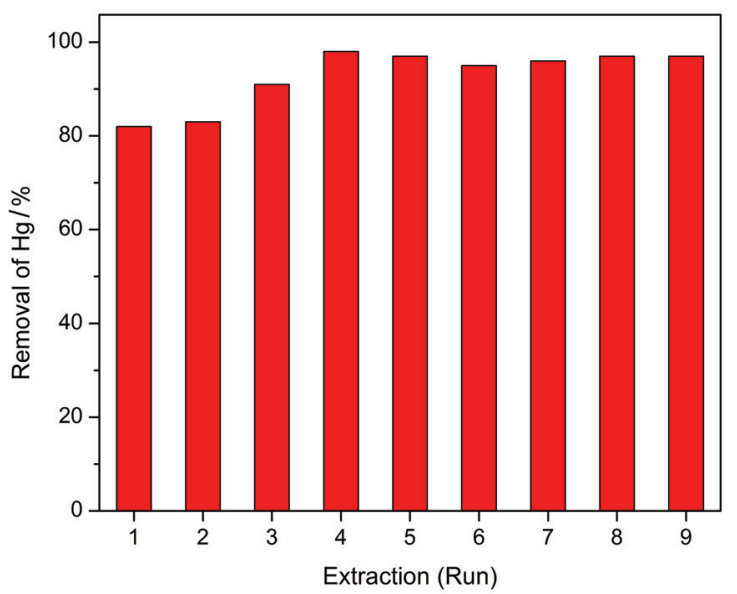

图 $3\left[\mathrm{C}_{8} \mathrm{MIM}_{\mathrm{P}} \mathrm{PF}_{6}\right.$ 循环萃取去除水中 $\mathrm{Hg}^{2+}: 50 \mathrm{~mL}, 1 \mathrm{mg} / \mathrm{L} \mathrm{Hg}^{2+}$ 水溶 液中加入 $1 \mathrm{~mL}\left[\mathrm{C}_{8} \mathrm{MIM}\right] \mathrm{PF}_{6}, 0.2 \mathrm{~mL}$ 1-甲基咪唑, $50{ }^{\circ} \mathrm{C}, 220 \mathrm{r} / \mathrm{min}$ 震荡 $2 \mathrm{~h}$; 离心分离, 去除上层水相; [ $\left.\mathrm{C}_{8} \mathrm{MIM}\right] \mathrm{PF}_{6}$ 中加入 $2 \mathrm{~mL}, 40 \%$ 甲酸溶 液, $50{ }^{\circ} \mathrm{C}, 220 \mathrm{r} / \mathrm{min}$ 震荡 $30 \mathrm{~min}$; 随后离心分离, 去除上层甲酸溶液,

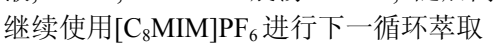

Figure 3 Assessment of the recycle ability of $\left[\mathrm{C}_{8} \mathrm{MIM}\right] \mathrm{PF}_{6}$ : Into $50 \mathrm{~mL}$ of $1 \mathrm{mg} / \mathrm{L} \mathrm{Hg}(\mathrm{II})$ solution was added $1 \mathrm{~mL}\left[\mathrm{C}_{8} \mathrm{MIM} \mathrm{PF}_{6}\right.$ containing $20 \%$ $(V / V)$ 1-methylimidazole, and the mixture was kept at $50{ }^{\circ} \mathrm{C}$ and shaken at $220 \mathrm{r} / \mathrm{min}$ for $2 \mathrm{~h}$. After centrifugation, into the separated $\left[\mathrm{C}_{8} \mathrm{MIM} \mathrm{PF}_{6}\right.$ was added $2 \mathrm{~mL} 40 \%$ formic acid solution, and the mixture was shaken at $50{ }^{\circ} \mathrm{C}$ for $30 \mathrm{~min}$. After that, the $\left[\mathrm{C}_{8} \mathrm{MIM}\right] \mathrm{PF}_{6}$ phase was collected for the next run

\section{References}

[1] Nolan, E. M.; Lippard, S. J. Chem. Rev. 2008, 108, 3443.

[2] Tchounwou, P. B.; Ayensu, W. K.; Ninashvili, N.; Sutton, D. Environ. Toxicol. 2003, 18, 149.

[3] Nriagu, J. O. Nature 1989, 338, 47.

[4] Onyido, I.; Norris, A. R.; Buncel, E. Chem. Rev. 2004, 104, 5911.

[5] Mureseanu, M.; Reiss, A.; Cioatera, N.; Trandafir, I.; Hulea, V. J. Hazard. Mater. 2010, 182, 197.

[6] Goyal, M.; Bhagat, M.; Dhawan, R. J. Hazard. Mater. 2009, 171, 1009.

[7] Zabihi, M.; Ahmadpour, A.; Asl, A. H. J. Hazard. Mater. 2009, 167, 230.

[8] Liu, J. F.; Zhao, Z. S.; Jiang, G. B. Environ. Sci. Technol. 2008, 42, 6949.

[9] Papaiconomou, N.; Lee, J. M.; Salminen, J.; Von Stosch, M.; Prausnitz, J. M. Ind. Eng. Chem. Res. 2007, 47, 5080.

[10] Germani, R.; Mancini, M. V.; Savelli, G.; Spreti, N. Tetrahedron Lett. 2007, 48, 1767.

[11] Visser, A. E.; Swatloski, R. P.; Reichert, W. M.; Mayton, R.; Sheff, S.; Wierzbicki, A.; Davis, J. H.; Rogers, R. D. Environ. Sci. Technol. 2002, 36, 2523 .

[12] Ersoz, M. Adv. Colloid Interface Sci. 2007, 134, 96.

[13] Gao, Z.; Ma, X. Anal. Chim. Acta 2001, 702, 50.

[14] Holbrey, J. D.; Visser, A. E.; Spear, S. K.; Reichert, W. M.; Swatloski, R. P.; Brokera, G. A.; Rogersa, R. D. Green Chem. 2003, 5 , 129.

[15] Jin, Z.; Xie, D. X.; Zhang, X. B.; Gong, Y. J.; Tan, W. Anal. Chem. 2012, 84, 4253 .

[16] Gkika, E.; Troupis, A.; Hiskia, A.; Papaconstantinou, E. Environ. Sci. Technol. 2005, 39, 4242.

[17] Ribeiro, A. S.; Vieira, M. A.; Willie, S.; Sturgeon, R. E. Anal. Bioanal. Chem. 2007, 388, 849.

[18] Gil, S.; Lavilla, I.; Bendicho, C. Ultrason. Sonochem. 2008, 15, 212.

[19] Liang, L. N.; Jiang, G. B.; Liu, J. F.; Hu, J. T. Anal. Chim. Acta 2003, 477, 131.

[20] Visser, A. E.; Swatloski, R. P.; Griffin, S. T.; Hartman, D. H.; Rogers, R. D. Sep. Sci. Technol. 2001, 36, 785.

[21] Chen, S.; Sun, H.; Zhong, Y.-S. J. Beijing University of Technology 2013, 39, 98. (陈莎, 孙浩, 钟嶷盛, 北京工业大学学报, 2013, 39, 98.)

[22] Rivas, B. L.; Jara, M.; Pereira, E. D. J. Appl. Polym. Sci. 2003, 89, 2852.

(Cheng, B.; Lu, Z.) 